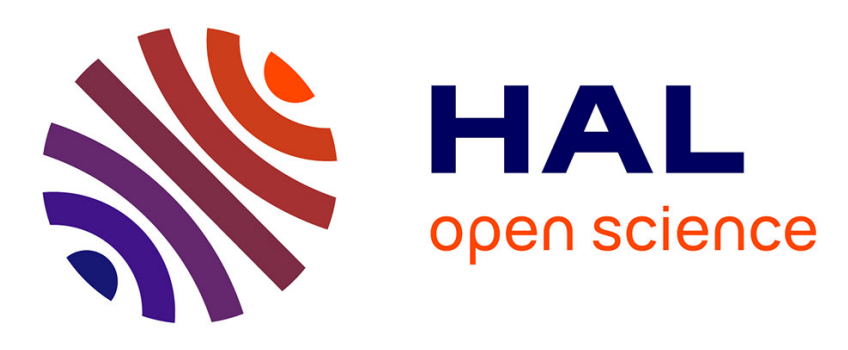

\title{
Phononic band-gap guidance of acoustic modes in photonic crystal fibers
}

\author{
V. Laude, A. Khelif, S. Benchabane, M. Wilm, T. Sylvestre, B. Kibler, \\ Arnaud Mussot, J.M. Dudley, H. Maillotte
}

\section{- To cite this version:}

V. Laude, A. Khelif, S. Benchabane, M. Wilm, T. Sylvestre, et al.. Phononic band-gap guidance of acoustic modes in photonic crystal fibers. Physical Review B: Condensed Matter and Materials Physics (1998-2015), 2005, 71, n 4, pp.045107. hal-00097432

\section{HAL Id: hal-00097432 \\ https://hal.science/hal-00097432}

Submitted on 26 Aug 2013

HAL is a multi-disciplinary open access archive for the deposit and dissemination of scientific research documents, whether they are published or not. The documents may come from teaching and research institutions in France or abroad, or from public or private research centers.
L'archive ouverte pluridisciplinaire HAL, est destinée au dépôt et à la diffusion de documents scientifiques de niveau recherche, publiés ou non, émanant des établissements d'enseignement et de recherche français ou étrangers, des laboratoires publics ou privés. 


\title{
Phononic band-gap guidance of acoustic modes in photonic crystal fibers
}

\author{
Vincent Laude, Abdelkrim Khelif, Sarah Benchabane, and Mikael Wilm \\ Département LPMO, Institut FEMTO-ST, CNRS UMR 6174, Université de Franche-Comté, 32 avenue de l'Observatoire, F-25044 \\ Besançon cedex, France \\ Thibaut Sylvestre, Bertrand Kibler, Arnaud Mussot, John M. Dudley, and Hervé Maillotte \\ Département d'Optique P. M. Duffieux, Institut FEMTO-ST, CNRS UMR 6174, Université de Franche-Comté, 16 route de Gray, \\ F-25030 Besançon cedex, France \\ (Received 16 September 2004; published 10 January 2005)
}

\begin{abstract}
The elastic modes guided along the axis of an optical fiber are obtained for an arbitrary finite cross section using waveguide finite element analysis. The band structure of acoustic phonons is obtained from this fullvector computation. The analysis is applied to the case of a photonic crystal fiber possessing a honeycomb lattice. It is shown that this fiber exhibits band gaps for elastic modes propagating along the longitudinal fiber axis. For frequencies within a band gap, the external boundary of the fiber becomes a defect of the phononic crystal that supports the propagation of guided elastic modes. Such boundary modes are very sensitive to the boundary conditions. The further introduction of a defect within the two-dimensional phononic crystal leads to the formation of highly confined elastic waveguide modes that copropagate in the same core volume as the guided optical mode. We consider the application of these properties to the suppression of stimulated Brillouin scattering and to enhanced collinear acousto-optical interactions. In particular, we obtain the optimum elastic modal shape that maximizes the acousto-optical scattering coefficient for given optical modes.
\end{abstract}

DOI: 10.1103/PhysRevB.71.045107 PACS number(s): 42.70.Qs, 42.65.Es, 63.20.-e, 78.20.Hp

\section{INTRODUCTION}

The study of wave propagation in microstructured and nanostructured materials is a subject of intense current research. Much attention has focused on electromagnetic wave propagation in photonic crystals ${ }^{1,2}$ and photonic crystal fibers (PCF) ${ }^{3}$ and in parallel on acoustic or elastic wave interactions in phononic crystals. ${ }^{4,5}$. From a fundamental viewpoint, both phononic and photonic crystals possess remarkable properties such as the existence of absolute band gaps that forbid the propagation of waves in any direction, which has already led to a number of important applications in both the photonics and acoustics fields. In particular, phononic and photonic crystals have been demonstrated to allow for very efficient mirrors, cavities and waveguides, acting on a wavelength scale.

In most previous works, the phononic and photonic properties of periodically structured materials have been considered independently, although the idea emerges that the interaction of photons and phonons within band-gap materials will lead to novel or enhanced effects, ${ }^{6-8}$ beyond traditional plane-wave acousto-optical interactions. However, the combination of phononic and photonic crystal structures has been addressed in the frame of a restricted dimensionality: enhanced photon-phonon interactions within one-dimensional acoustic band gaps have been considered ${ }^{6,8}$ and, for the case of PCFs, phononic band gaps have been demonstrated in a preform. ${ }^{7}$ The latter situation, analyzed by Rayleigh ${ }^{7}$ and finite-difference time domain ${ }^{9}$ (FDTD) methods, was restricted to two-dimensional in-plane band gaps (i.e., for a zero longitudinal acoustic wavevector) that give rise to static elastic modes confined across the transverse fiber cross section. The acousto-optical interaction in the case of static elastic modes can be employed to modulate dynamically the phase of an optical beam, ${ }^{7}$ however, it cannot give rise to optical mode coupling through acousto-optical diffraction. The more general consideration of acousto-optical diffraction then requires us to increase the dimensionality of the problem by performing a global analysis of phononic-band-gap modes. This is the purpose of the present paper, which considers the full three-dimensional problem. More precisely, we focus on the PCF case, in the aim of showing it can also be considered a phononic crystal fiber.

Recently, the out-of-plane acoustic band gaps in a twodimensional solid-solid phononic crystal were investigated by a plane-wave expansion (PWE) approach. ${ }^{10}$ The appearance of elastic modes guided along a defect of the twodimensional solid-solid phononic crystal was further demonstrated. ${ }^{11}$ However, the PWE method ${ }^{12}$ followed in these works can not be applied directly in the case of a finite PCF cross section, since it assumes either an infinite structure or periodic boundary conditions. Furthermore, the PWE method applies to a solid-solid composition, whereas a PCF is a periodic arrangement of micron-size cylindrical parallel hollow holes inside a silica matrix. We make use in this work of a particular formulation of the finite element method (FEM), the waveguide FEM, ${ }^{13}$ that is suited to the description of the propagation of elastic modes guided along a cylindrical fiber. With this technique, the waveguide modes can be obtained by imposing a longitudinal wave vector and solving for the discrete eigenfrequencies. By this procedure the band structure for acoustic modes is obtained.

For illustration purposes we consider the case of a PCF with a honeycomb lattice, forming a two-dimensional phononic crystal. We observe the existence of out-of-plane phononic band gaps in the PCF and exploit this property for strongly confining elastic waveguide modes that propagate along the longitudinal fiber axis. We further verify that the 


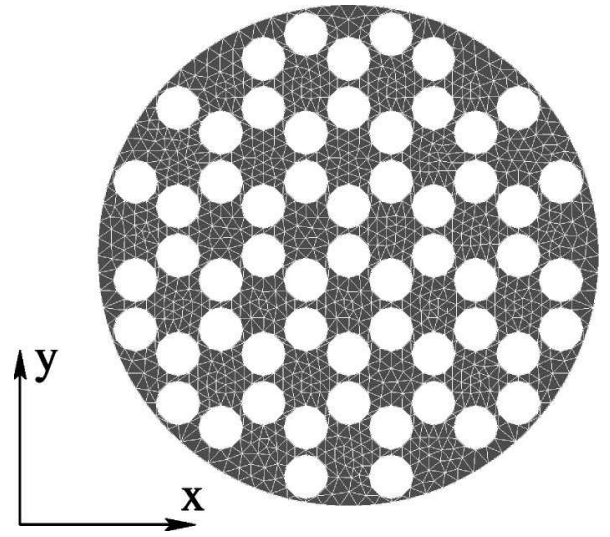

FIG. 1. Example of a finite element mesh of a honeycomb photonic crystal fiber.

PCF supports single-mode optical guidance, though by modified total internal reflection rather than by a photonic crystal effect. This yields insights into the physics and properties of microstructures and nanostructures supporting phononic-band-gap guidance of elastic modes while simultaneously presenting single-mode optical guidance in the same PCF core region. Purposely, we then discuss particular design examples that could lead to the hypersonic band-gap inhibition of stimulated Brillouin scattering and to enhanced acousto-optical interactions by energy confinement. Design rules for optimizing or conversely minimizing the elastooptical scattering coefficient are drawn.

\section{ELASTIC WAVEGUIDE MODES}

A PCF is typically based on a periodic arrangement of micron-size cylindrical parallel holes inside a silica matrix, with a central defect acting as a core. Light is guided along the hollow or solid core either by a photonic-band-gap effect $^{14}$ or by modified total internal reflection, ${ }^{15}$ respectively. As regards the propagation of elastic waves, the elastic energy vanishes within the hollow cylinders and is thus constrained to remain within the silica. The boundaries of the hollow cylinders can be considered as free from tractions and act as very efficient scatterers for elastic waves of any polarization. Silica is an isotropic material for the propagation of elastic waves.

Our analysis of the elastic modes is based on the waveguide finite element method (FEM) which combines a planewave-like ansatz for modes along the assumed infinite propagation direction with a finite element approach that is advantageous in allowing the modeling of arbitrary cross sections. ${ }^{13}$ With this technique, the two-dimensional waveguide section in the plane $(x, y)$ is meshed using finite elements, and the displacements are represented by piecewise polynomials within the elements. Along the propagation direction $z$ (aligned with the PCF axis) a sinusoidal variation of the displacements is imposed with a given wave vector $k$. To account for propagation along the $z$ axis, a harmonic dependence $\exp [j(\omega t-k z)]$ is considered. Figure 1 displays an example of a mesh used in this work to represent the cross section of a PCF. The finite elements are triangles with three vertices and six nodes, that is one node at each vertex plus one node at the center of each edge. The mesh of Fig. 1 includes 1856 finite elements and a total of 4441 nodes. The unknowns, or degrees of freedom (DOF), are the three displacements at each node which amounts to a total of 13323 DOF. Interpolating polynomials of degree 2 in the intrinsic coordinates of the triangle are used.

For isotropic materials and a cylindrical geometry, the transverse components $u_{x}$ and $u_{y}$ and the longitudinal component $u_{z}$ of the displacements are in phase quadrature. In order to guarantee a unique solution to the variational problem associated to the finite element method, we use the realvalued formulation within each finite element

$$
\begin{aligned}
& u_{x}(x, y, z ; t)=\mathbf{p}(x, y)^{T} \cdot \hat{\mathbf{u}}_{x} \cos (\omega t-k z), \\
& u_{y}(x, y, z ; t)=\mathbf{p}(x, y)^{T} \cdot \hat{\mathbf{u}}_{y} \cos (\omega t-k z), \\
& u_{z}(x, y, z ; t)=\mathbf{p}(x, y)^{T} \cdot \hat{\mathbf{u}}_{z} \sin (\omega t-k z),
\end{aligned}
$$

where $\hat{\mathbf{u}}=\left(\hat{\mathbf{u}}_{x}, \hat{\mathbf{u}}_{y}, \hat{\mathbf{u}}_{z}\right)^{T}$ is the vector of the $3 n$ displacements at the $n$ nodes of the finite element and the $\mathbf{p}$ is a vector of $n$ Lagrange interpolation polynomials $(n=6$ in all computations in this work). The dynamics of elastic waves are obtained as the solution of a variational problem involving the kinetic and strain energies. The kinetic energy in a onewavelength-long finite element with section $\sigma$ is

$$
\mathcal{K}=\omega^{2} \int_{0}^{2 \pi / k} d z \int_{\sigma} d x d y \mathbf{u}^{T} \cdot \rho \cdot \mathbf{u},
$$

where $\rho$ is the mass density and the displacement vector $\mathbf{u}=\left(u_{x}, u_{y}, u_{z}\right)^{T}$. Inserting Eqs. (1)-(3) and integrating along $z$ yields

$$
\mathcal{K}=\frac{\omega^{2}}{2 k}\left(\hat{\mathbf{u}}^{T} \cdot M_{\sigma} \cdot \hat{\mathbf{u}}\right),
$$

with the elementary mass matrix $M_{\sigma}$ and the polynomial matrix $P$ defined by

$$
M_{\sigma}=\int_{\sigma} d x d y P^{T} \cdot \rho \cdot P,
$$

and

$$
P=\left(\begin{array}{ccc}
\mathbf{p}^{T} & 0 & 0 \\
0 & \mathbf{p}^{T} & 0 \\
0 & 0 & \mathbf{p}^{T}
\end{array}\right) .
$$

Matrices $M_{\sigma}$ and $P$ have dimensions $3 n \times 3 n$ and $3 \times 3 n$, respectively. Note that the considered finite element is implicitly three dimensional, although only its two-dimensional cross section needs to be specified; its length along the $z$ axis is exactly one wavelength. Similarly, the strain energy within the finite element is

$$
\mathcal{U}=\int_{0}^{2 \pi / k} d z \int_{\sigma} d x d y \mathbf{S}^{T} \cdot c \cdot \mathbf{S},
$$

where the strain tensor $S$ and the elastic tensor $c$ are written in contracted (or Voigt) notation, i.e., $c$ is a $6 \times 6$ matrix and 
$\mathbf{S}$ is a six-component vector. Inserting Eqs. (1)-(3), integrating along $z$ and making use of sine and cosine orthogonality, the strain energy can be expressed as

$$
\mathcal{U}=\frac{1}{2 k}\left(\hat{\mathbf{u}}^{T} \cdot K_{\sigma} \cdot \hat{\mathbf{u}}\right),
$$

with the elementary stiffness matrix

$$
K_{\sigma}=\int_{\sigma} d x d y\left(A_{r}^{T} \cdot c \cdot A_{r}+A_{i}^{T} \cdot c \cdot A_{i}\right),
$$

where

$$
\begin{aligned}
A_{r} & =\left(\begin{array}{ccc}
\mathbf{p}_{, x}^{T} & 0 & 0 \\
0 & \mathbf{p}_{, y}^{T} & 0 \\
0 & 0 & k \mathbf{p}^{T} \\
\mathbf{p}_{, y}^{T} & \mathbf{p}_{, y}^{T} & 0 \\
0 & 0 & 0 \\
0 & 0 & 0
\end{array}\right), \\
A_{i} & =\left(\begin{array}{ccc}
0 & 0 & 0 \\
0 & 0 & 0 \\
0 & 0 & 0 \\
0 & 0 & 0 \\
0 & -k \mathbf{p}^{T} & \mathbf{p}_{, y}^{T} \\
-k \mathbf{p}^{T} & 0 & \mathbf{p}_{, x}^{T}
\end{array}\right),
\end{aligned}
$$

and the vectors $\mathbf{p}_{x}^{T}=\left(\partial p_{1} / \partial x, \ldots, \partial p_{n} / \partial x\right)$ and $\mathbf{p}_{, y}^{T}=\left(\partial p_{1} / \partial y, \ldots, \partial p_{n} / \partial y\right)$. The matrix $K_{\sigma}$ has dimensions $3 n \times 3 n$ while matrices $A_{r}$ and $A_{i}$ have dimensions $6 \times 3 n$.

As is usual with elastic FEM problems, the solution of the variational problem with no external applied forces is given by the linear equation

$$
\left(K(k)-\omega^{2} M\right) \hat{\mathbf{u}}=0,
$$

where the stiffness matrix $K(k)$ and the mass matrix $M$ are obtained by assembling the elementary stiffness and mass matrices respectively by standard procedures. ${ }^{16} K(k)$ is actually a second order matrix polynomial in $k$, as can be seen from Eqs. (10)-(12), while $M$ is $k$ independent. Equation (13) is in the form of a generalized eigenvalue problem for $\omega^{2}$ if $k$ is considered a parameter. The corresponding eigenmodes are the elastic waveguide modes of the fiber, or alternatively acoustic phonons. It is worth noting that for all eigenmodes, the kinetic and strain energies are equal. In fact they each amount to one half of the total mode energy. As a test for the waveguide FEM, we first verified that we were able to obtain the elastic modes guided by a plain cylinder with a circular cross section, for which analytical solutions are known in connection with the Pochhammer-Chree dispersion relations. ${ }^{17}$

We have then used the waveguide FEM technique to analyze the phononic band-gap characteristics of the silica-air honeycomb structures shown in the meshed cross section as insets in Fig. 2. Note that here, as elsewhere in this paper, open circles are associated with holes. We consider in Fig. 2
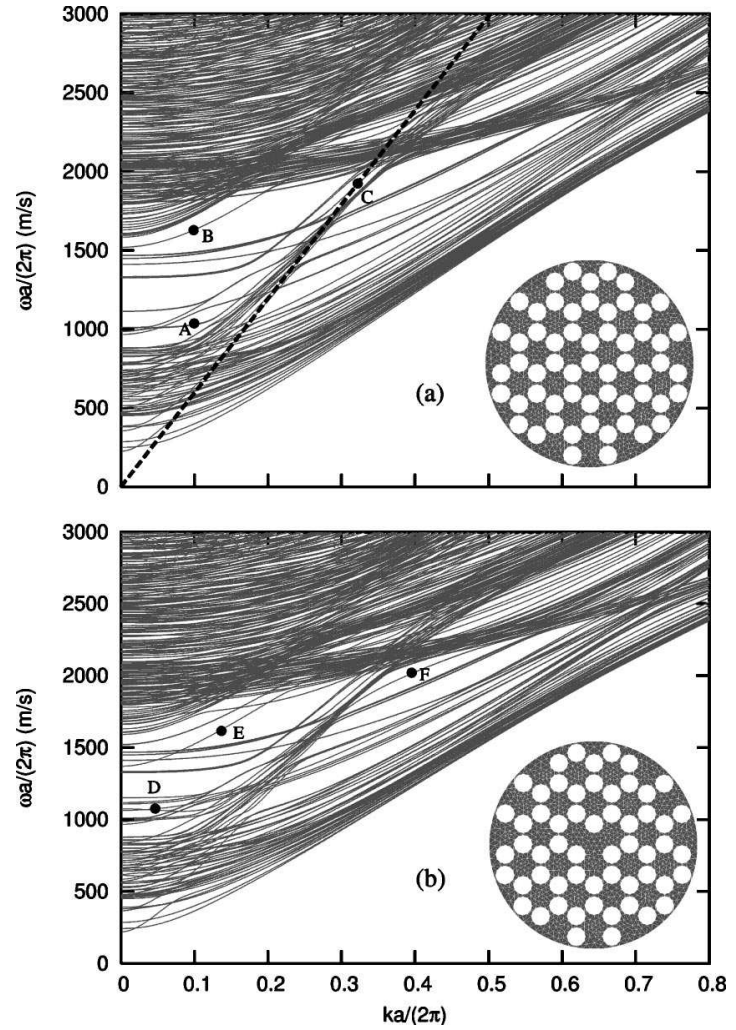

FIG. 2. Band structures for elastic waveguide modes of honeycomb silica photonic crystal fibers (a) without and (b) with a central defect. The two-dimensional meshes of the cross-sections are shown as insets. The longitudinal line in silica is shown in (a).

honeycomb structures both (a) without and (b) with a central solid silica defect. For this structure having a hole pitch (center-to-center distance) of $a$, the PCF diameter is then approximately $10 a$. Band structures are obtained by solving for the generalized eigenvalue problem of Eq. (13) as a function of the longitudinal wave vector $k$. It can be remarked that the static elastic modes confined across the transverse fiber cross section, considered, e.g., in Refs. 7 and 9, are obtained for $k=0$. Using proper finite elements, both the stiffness and mass matrices are positive definite, which ensures the positivity of the eigenvalues. We use a Cholesky factorization of the stiffness matrix to transform the generalized eigenvalue problem into a standard one, followed by a Lanczös algorithm to obtain the lowest eigenvalues. The band structure in Fig. 2(a) for elastic modes without a central defect exhibits high density except in several regions where only isolated branches exist. An examination of the corresponding eigenvectors reveals that in the dense regions the elastic modes are similar to those of a solid cylinder. In particular, their energy density is spread in the whole fiber. In contrast, isolated branches correspond to modes that are confined to the external boundary of the PCF, which is clamped in the calculation. When the external boundary is considered stress-free instead, modes in the dense regions are only very slightly affected, but isolated branches are displaced. We interpret the isolated branches as corresponding to surface modes that are trapped along the external boundary of the PCF when the frequency falls within a phononic band gap. 

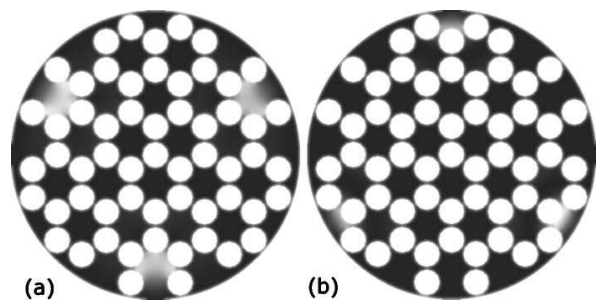

FIG. 3. Energy density (shown in gray-scale) of guided elastic modes propagating along the external boundary of the photonic crystal fiber of Fig. 2(a) for the points labeled (a) $A$ and (b) $B$ in the band structure of Fig. 2(a).

Indeed, in this case the waves cannot penetrate deeply the inner honeycomb structure and the external boundary acts as a defect of the phononic crystal that supports highly confined modes. Figure 3 displays the energy distribution of several such phononic crystal boundary modes.

Considering now the phononic band-gap properties of the structure shown in Fig. 2(b), which is identical to the perfect crystal structure in Fig. 2(a) except for an additional central silica defect, we expect confined elastic modes to appear within the bandgaps. A comparison of the band structures of Figs. 2(a) and 2(b) reveals that the densely populated mode regions, as well as the isolated branches corresponding to modes confined between the external boundary and the honeycomb-structured interior, are very similar, indicating insensitivity of these modes to the presence of the core defect. However, it is significant that additional isolated branches appear within the bandgaps in Fig. 2(b), and an examination of modes along these particular branches reveals that they are confined and trapped within the silica defect. Figure 4 illustrates the energy distribution of several of these core modes. Their waveguiding clearly relies on the out-of-plane elastic band-gap properties of the PCF. It is also significant that some of these core modes can exhibit surprising dispersion relations. For instance, the mode depicted in Fig. 4(a) [point $D$ in Fig. 2(b)] has a zero group velocity for some value of the $k$ wave vector.

\section{ELASTO-OPTICAL INTERACTIONS}

Beam propagation method (BPM) simulations were used to check that optical core guidance is possible under realistic
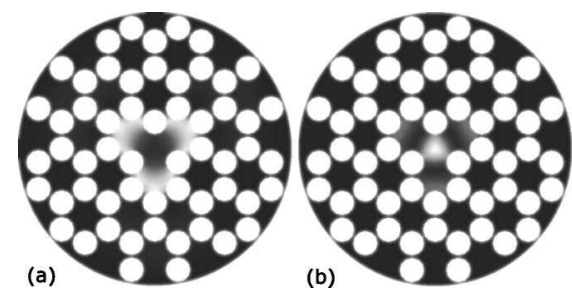

(b)

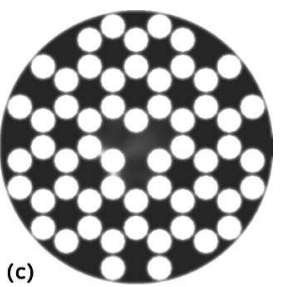

FIG. 4. Energy density (shown in gray-scale) of guided elastic modes propagating in the core of the photonic crystal fiber of Fig. 2(b) by a phononic band-gap effect for the points labeled (a) $D$, (b) $E$, and (c) $F$ in the band structure of Fig. 2(b).

conditions for the PCF with a central core defect depicted in Fig. 2(b). Figure 5(a) and 5(b) show the fundamental optical TE and TM modes at $1.55 \mu \mathrm{m}$, for a hole diameter and pitch of $1.01 \mu \mathrm{m}$ and $1.13 \mu \mathrm{m}$, respectively. With such dimensions, the frequency of the acoustic waveguide mode of Fig. 4(b), corresponding to point $(E)$ in Fig. 2(b), is $1.3 \mathrm{GHz}$. Hence, from Figs. 4 and 5, we anticipate that guided acoustic modes within such an out-of-plane phononic band-gap structure will enable enhanced collinear acousto-optical interactions, presenting both a significantly increased interaction length compared to in-plane acousto-optical interactions across the PCF cross section ${ }^{7}$ and the possibility of coherently coupling and transferring energy between several optical modes.

Clearly, in the usual case of plane-wave acousto-optical interaction in a bulk material, the acoustic or elastic waves are generated by an extended plane transducer and it is difficult to match the optical and acoustic modal shapes. The possibility of copropagating optical and elastic energy along the same fiber core with tight confinement is then intuitively appealing in view of enhancing elasto-optical interactions. For definiteness, we consider the elasto-optical diffraction coefficient to be proportional to

$$
\kappa=\int_{\sigma} d x d y E_{i}^{(1)} E_{j}^{(2)} p_{i j k l} S_{k l},
$$

where $\mathbf{E}^{(1)}$ and $\mathbf{E}^{(2)}$ are the (real-valued) electric field vectors of the incident and the scattered optical modes, respectively, $S$ is the strain tensor associated to the elastic mode, and $p$ is the elasto-optical tensor. In this expression, and from this

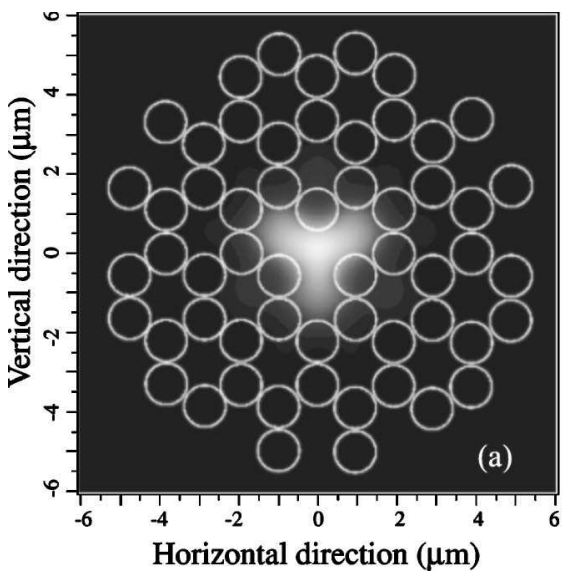

Horizontal direction $(\mu \mathrm{m})$

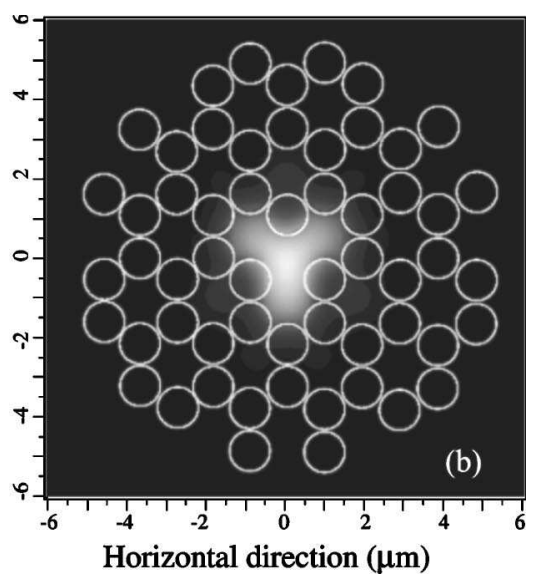

$045107-4$
FIG. 5. Optical (a) TE and (b) TM mode simulations in the same photonic crystal fiber as in Fig. 4. 

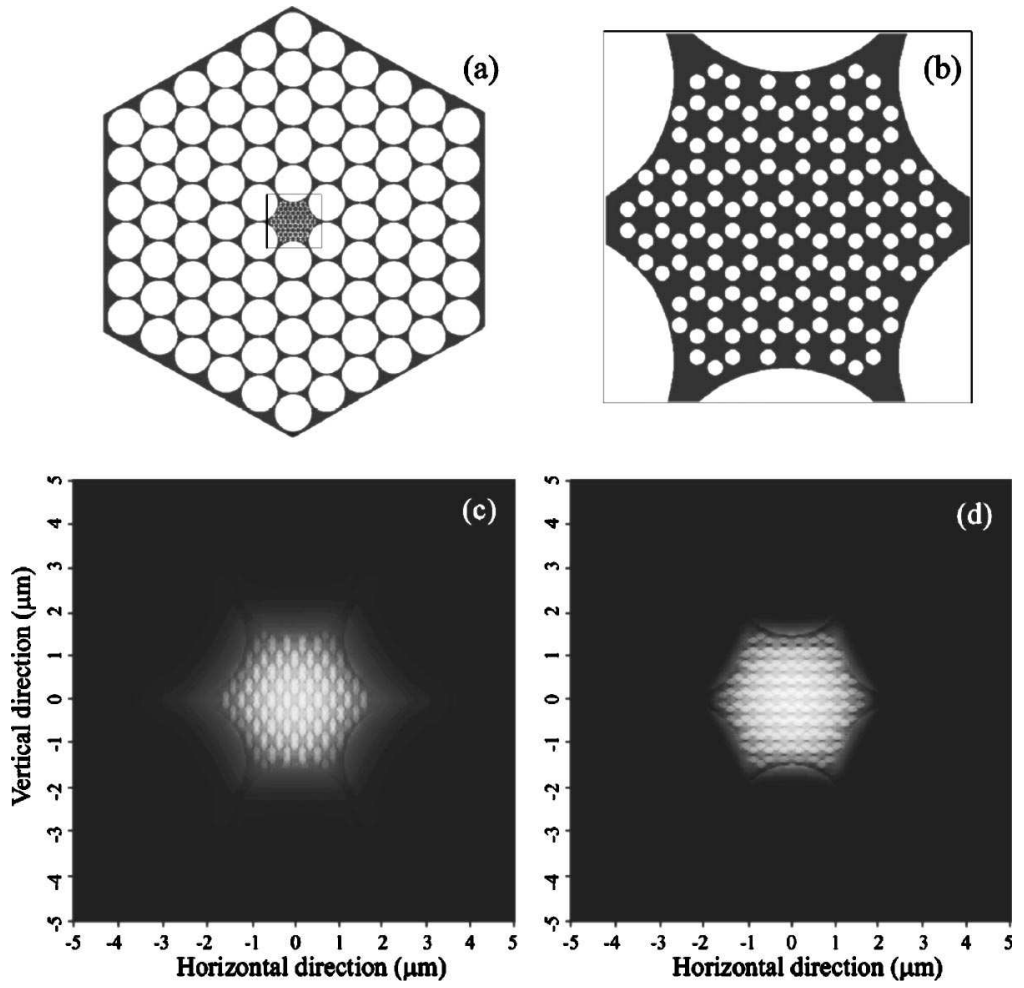

FIG. 6. (a) Photonic crystal fiber cross section showing a combined microstructure and nanostructure designed for simultaneous core optical guidance and the inhibition of stimulatedBrillouin-scattering phonon propagation. (b) Detail of the core region. (c) and (d) Numerical simulations of the fundamental TE and TM optical modes at $1.55 \mu \mathrm{m}$, respectively. point on, we use full tensorial notation and the repeated index summation convention; indices $i, j, k, l=1,2,3$. We further rewrite the strain energy of Eq. (8) as

$$
\mathcal{U}=\frac{2 \pi}{k} \int_{\sigma} d x d y S_{i j} c_{i j k l} S_{k l}
$$

and notice that the elastic energy is twice this quantity. We wish to identify the elastic modal shape that will maximize $\kappa$ for given optical modes and a fixed elastic energy, for instance unity. This can be accomplished by using the Lagrange multiplier method. Introducing the Lagrange multiplier $\xi$, we look for an extremum of the functional $\kappa-\xi \mathcal{U}$, considered a function of $S_{i j}$, and then identify $\xi$ by the condition $\mathcal{U}=1$. The result is that the elastic stress tensor associated with the elastic mode, $T_{i j}=c_{i j k l} S_{k l}$ must be

$$
T_{i j}=A p_{i j k l} E_{k}^{(1)} E_{l}^{(2)},
$$

where $A$ is some proportionality constant. It might be difficult in practice to achieve an elastic modal shape given exactly by the optimal shape of Eq. (16). However, even a partial matching of optical and elastic modes will clearly result in an improved elasto-optical diffraction coefficient. Among the three elastic core modes shown in Fig. 4, the mode labeled $(E)$ has the largest elasto-optical diffraction coefficient in combination with the TE or TM optical modes displayed in Fig. 5. By controlling the anisotropy of the PCF, e.g., through the anisotropic distribution of holes, anisotropic acousto-optical interaction between optical modes of different polarization should also be possible. Such an interaction may, for instance, find applications in ultrashort laser pulse shaping, as an alternative to bulk acousto-optical programmable filters. ${ }^{18}$
As another possible application of elasto-optical interaction in a PCF, we suggest that the design of a proper nanostructuration of the fiber could lead to the inhibition of stimulated Brillouin scattering (SBS), or at least to an increase in the SBS threshold, by engineering a phononic band gap such that the phonons involved in SBS are not allowed to propagate any more. This is, for instance, of much practical significance around the technologically important wavelength range around $1550 \mathrm{~nm}$ as SBS is highly detrimental for fiber communications systems. ${ }^{19}$

SBS is a three-wave nonlinear interaction in which an intense, incident optical pump wave of frequency $\omega_{p}$ is backscattered into a downshifted Stokes wave of frequency $\omega_{s}=\omega_{p}-\omega$ through the coherent generation of an acoustic phonon at frequency $\omega$ via material electrostriction. ${ }^{20}$ The scattered acoustic phonons modulate the refractive index of the medium, acting as a Bragg grating propagating forward at the longitudinal acoustic velocity $v$, so that the reflected optical mode is downshifted through the Doppler effect. The longitudinal wave vector of the SBS phonon is given by the phase matching condition, $k=k_{p}-k_{s}$, where $k_{p}$ and $k_{s}$ are the optical pump and Stokes wave vectors, and $\omega=v k$ defines the SBS phonon dispersion relation. In silica, $v=5970 \mathrm{~m} / \mathrm{s}$, which is much smaller than the speed of light, so that the acoustic wave vector and the SBS hypersound frequency are very well approximated in single-mode fibers by $k=2 k_{p}$ and then $\omega=2 n v \omega_{p} / c$, where $n$ is the effective index of the optical mode. Using typical parameters for telecommunication fibers at $1.55 \mu \mathrm{m}$, the acoustic wavelength and frequency are respectively $543 \mathrm{~nm}$ and $11 \mathrm{GHz}$. In general, optically guiding high air-fill fraction PCFs will be acoustically multimode for such small acoustic wavelengths, but if a central additional nanostructure is added in the PCF core region to open an out-of-plane phononic band gap for the phonon couple 
$(\omega, k)$, the coherent amplification of SBS phonons will be inhibited at these frequencies.

To illustrate this more explicitly, the straight line in Fig. 2(a) passing through the origin shows the SBS phonon dispersion relation. Point $\mathrm{C}$ in Fig. 2(a) corresponds to $\omega a /(2 \pi)=1911 \mathrm{~m} / \mathrm{s}$ and $\mathrm{ka} /(2 \pi)=0.32$. Although it appears from the figure that the nanostructure opens up only a partial out-of-plane band gap, the band-gap width is in fact significantly larger than the SBS linewidth. In particular, we consider the mixed microstructure-nanostructure PCF structure in Figs. 6(a) and 6(b). Here, the hole diameter and pitch are 2.59 and $2.76 \mu \mathrm{m}$, respectively, for the exterior microstructure (typical solid core PCF dimensions) and 145 and $207 \mathrm{~nm}$, respectively, for the internal honeycomb nanostructure (technologically feasible sizes ${ }^{3}$ ). The exterior PCF microstructure is designed such that highly confined optical guidance in the core is obtained. The nanostructure dimensions in the core are chosen such that SBS phonons fall within a phononic band gap. Optical guidance in such a structure was verified using standard beam propagation method vector simulations, and Figs. 6(c) and 6(d) show the guided mode solutions for the TE (horizontal) and TM (vertical) electric field components. Efficient optical guidance at $1.55 \mu \mathrm{m}$ is obtained in the presence of the phononic nanostructure. Calculation of the associated effective indices ( $n=1.2046$ and $n=1.1557$, respectively) allows us to verify that the phononic band-gap is opened about a mean SBS frequency of $9.3 \mathrm{GHz}$ for both polarizations.

We note that, contrary to current modulation techniques used to suppress SBS in optical fibers that often impair the overall transmission performance, the proposed nanostructuration of a PCF is totally passive. However, a precise prediction of the increase in the SBS threshold that can be achieved using such a nanostructured core requires a more complete model of SBS than used in this work. In particular, the effect of electrostriction on the coupling between phonons and photons, considered as an elementary coupling mechanism for SBS, should be considered. However, electrostriction will have little or no effect on photonic and phononic spectra (band diagrams) as presented in the present work, and these can be used as the basis of a coupled-mode theory of SBS.

\section{CONCLUSION}

In summary, we have used a waveguide finite element method to obtain the elastic modes guided by a photonic crystal fiber consisting of microscopic or nanoscopic air holes in silica. The method yields modes of any polarization and applies for arbitrary cross sections. We have demonstrated that out-of-plane phononic band gaps exist in a photonic crystal fiber with a honeycomb lattice, raising the possibility of guiding elastic modes confined along the external boundary of the PCF as well as inside a defect of the phononic crystal. Based on these features, the hybrid guidance of acoustic and optical guided modes has been demonstrated. These observations yield insights into the possibility of enhancing acousto-optical interactions as well as inhibiting phonons induced by the stimulated Brillouin scattering effect.
${ }^{1}$ E. Yablonovitch, Phys. Rev. Lett. 58, 2059 (1987).

${ }^{2}$ S. John, Phys. Rev. Lett. 58, 2486 (1987).

${ }^{3}$ P. S. J. Russell, Science 299, 358 (2003).

${ }^{4}$ M. M. Sigalas and E. N. Economou, Solid State Commun. 86, 141 (1993).

${ }^{5}$ M. S. Kushwaha, P. Halevi, L. Dobrzynski, and B. DjafariRouhani, Phys. Rev. Lett. 71, 2022 (1993).

${ }^{6}$ J. M. Worlock and M. L. Roukes, Nature (London) 421, 802 (2003).

${ }^{7}$ P. S. J. Russell, E. Marin, A. Diez, S. Guenneau, and A. B. Movchan, Opt. Express 20, 2555 (2003).

${ }^{8}$ M. M. de Lima, Jr., R. Hey, and P. V. Santos, Appl. Phys. Lett. 83, 2997 (2003).

${ }^{9}$ A. Khelif, B. Djafari-Rouhani, V. Laude, and M. Solal, J. Appl. Phys. 94, 7944 (2004).

${ }^{10}$ M. Wilm, A. Khelif, S. Ballandras, V. Laude, and B. DjafariRouhani, Phys. Rev. E 67, 065602(R) (2003).

${ }^{11}$ A. Khelif, M. Wilm, V. Laude, S. Ballandras, and B. DjafariRouhani, Phys. Rev. E 69, 067601 (2004).
${ }^{12}$ M. Wilm, S. Ballandras, V. Laude, and T. Pastureaud, J. Acoust. Soc. Am. 112, 943 (2002).

${ }^{13}$ B. Aalami, J. Appl. Mech. 40, 1067 (1973).

${ }^{14}$ R. F. Cregan, B. J. Mangan, J. C. Knight, T. A. Birks, P. S. J. Russell, P. J. Roberts, and D. C. Allan, Science 285, 1537 (1999).

${ }^{15}$ T. A. Birks, J. C. Knight, and P. S. Russell, Opt. Lett. 22, 961 (1997).

${ }^{16}$ O. C. Zienkiewicz and R. L. Taylor, Finite Element Method (Butterworth Heinemann, London, 2000).

${ }^{17}$ D. Royer and E. Dieulesaint, Elastic Waves in Solids (Wiley, New York, 1999), Vol. 1.

${ }^{18}$ F. Verluise, V. Laude, Z. Cheng, C. Spielmann, and P. Tournois, Opt. Lett. 25, 575 (2000).

${ }^{19}$ G. P. Agrawal, Nonlinear Fiber Optics (Academic, New York, 2001).

${ }^{20}$ R. Y. Chiao, C. H. Townes, and B. P. Stoicheff, Phys. Rev. Lett. 12, 592 (1964). 